\title{
New holographic reconstruction of scalar-field dark-energy models in the framework of chameleon Brans-Dicke cosmology
}

\author{
Surajit Chattopadhyay ${ }^{1, \mathrm{a}}$, Antonio Pasqua ${ }^{2, \mathrm{~b}}$, Martiros Khurshudyan ${ }^{3,4, \mathrm{c}}$ \\ ${ }^{1}$ Pailan College of Management and Technology, Bengal Pailan Park, Kolkata 700 104, India \\ ${ }^{2}$ Department of Physics, University of Trieste, Via Valerio, 2, 34127 Trieste, Italy \\ ${ }^{3}$ Department of Theoretical Physics, Yerevan State University, 1 A. Manookian, Yerevan, Armenia \\ ${ }^{4}$ Present address: Max Planck Institute of Colloids and Interfaces, Potsdam-Golm Science Park, Am Mühlenberg 1 OT Golm, 14476 Potsdam, \\ Germany
}

Received: 12 June 2014 / Accepted: 16 September 2014 / Published online: 27 September 2014

(c) The Author(s) 2014. This article is published with open access at Springerlink.com

\begin{abstract}
Motivated by the work of Yang et al. (Mod. Phys. Lett. A 26:191, 2011), we report on a study of the new holographic dark energy (NHDE) model with energy density given by $\rho_{D}=\frac{3 \phi^{2}}{4 \omega}\left(\mu H^{2}+v \dot{H}\right)$ in the framework of chameleon Brans-Dicke cosmology. We have studied the correspondence between the quintessence, the DBI-essence, and the tachyon scalar-field models with the NHDE model in the framework of chameleon Brans-Dicke cosmology. Deriving an expression of the Hubble parameter $H$ and, accordingly, $\rho_{D}$ in the context of chameleon Brans-Dicke chameleon cosmology, we have reconstructed the potentials and dynamics for these scalar-field models. Furthermore, we have examined the stability for the obtained solutions of the crossing of the phantom divide under a quantum correction of massless conformally invariant fields, and we have seen that the quantum correction could be small when the phantom crossing occurs and the obtained solutions of the phantom crossing could be stable under the quantum correction. It has also been noted that the potential increases as the matterchameleon coupling gets stronger with the evolution of the universe.
\end{abstract}

\section{Introduction}

The approaches to account for the late-time cosmic acceleration, which is suggested by the two independent observational signals on distant Type Ia Supernovae (SNeIa) [1$3]$, the cosmic microwave background (CMB) temperature anisotropies measured by the WMAP and Planck satellites

\footnotetext{
a e-mail: surajcha@iucaa.ernet.in; surajitchatto@outlook.com

b e-mail: toto.pasqua@gmail.com

c e-mail: khurshudyan@yandex.ru
}

[4-6] and Baryon Acoustic Oscillations (BAO) [7,8], fall into two representative categories: in the first, the concept of "dark energy" is introduced in the right-hand side of the Einstein equation in the framework of general relativity (for good reviews see [9-11]), while in the second one the lefthand side of the Einstein equation is modified, leading to a modified gravitational theory (which is well reviewed in [1215]). In a recent review, Bamba et al. [10] demonstrated that both dark-energy models and modified gravity theories seem to be in agreement with data and hence, unless higher precision probes of the expansion rate and the growth of structure will be available, these two rival approaches could not be discriminated. The physical origin of dark energy (DE) is one of the largest mysteries not only in cosmology but also in fundamental physics $[9,16-19]$. The cosmological constant $\Lambda$ represents the earliest and the simplest theoretical candidate proposed in order to explain the observational evidence of accelerated expansion. Some tentative deviations from the $\Lambda \mathrm{CDM}$ model may eventually rule out an exact cosmological constant [20,21]. A considerable number of models for DE have been proposed up to now to explain the late-time cosmic acceleration without the cosmological constant. Such models include a canonical scalar field, the so-called quintessence, a non-canonical scalar field such as phantom, tachyon scalar field motivated by string theories, and a fluid with a special equation of state (EoS) called a Chaplygin gas. Other well studied candidates for $\mathrm{DE}$ are the k-essence, the quintom and the agegraphic dark energy (ADE) models. Studies on the models previously mentioned include $[9,10,22$ 29]. There also exists a proposal known as holographic dark energy (HDE) proposed by Li [30], following the idea that the short distance cut-off is related to the infrared cut-off and it was assumed in [30] that the infrared cut-off relevant to the dark energy is the size of the event horizon. Some notable works on HDE include [31-34]. Furthermore, there exists 
plethora of literature on HDE in theoretical aspects as well as observational constraints e.g. [35-37].

The equation of state $(\mathrm{EoS})$ parameter, defined as $w_{\mathrm{DE}}=$ $p_{\mathrm{DE}} / \rho_{\mathrm{DE}}$ (where $p_{\mathrm{DE}}$ and $\rho_{\mathrm{DE}}$ denote the pressure and density of DE, respectively), is one of the most important quantity used to describe the features of DE models. If we restrict ourselves to four-dimensional Einstein's gravity, almost all DE models can be classified according to the behavior of the EoS parameter as follows [38]: (i) Cosmological constant: $w_{\Lambda}=-1$; (ii) Quintessence: $w_{Q} \geqslant-1$; (iii) Phantom: $w_{P} \leqslant-1$ and (iv) Quintom: its EoS is able to evolve across the cosmological constant boundary. Scalar field models of dark energy are among the most promising and best elaborated ones to match observations of the accelerated expansion of the Universe. The phantom-like behavior of $w_{\mathrm{DE}}$ may appear from Brans-Dicke (BD) scalar-tensor gravity, from non-standard (negative) potentials, from the non-minimal coupling of a scalar Lagrangian with gravity, or even the usual matter may appear in phantom-like form [26]. Studies devoted to phantom cosmology and to quintessence include [39-44] and [45-47], respectively.

In this contribution, we are concerned with a new holographic reconstruction of scalar-field dark-energy models in the framework of chameleon Brans-Dicke cosmology. In the context of the cosmological reconstruction problem, some notable contributions are [48-50]. The current work is primarily motivated by [51] and also got inspiration from $[52,53]$. It has already been stated that HDE model is based on the holographic principle, according to which the number of degrees of freedom of a physical system scales with the area of its boundary [31]. Although HDE gives the observational value of $\mathrm{DE}$ in the universe and can drive the universe to an accelerated expansion phase, an obvious drawback concerning causality appears in this proposal [51]. In view of this limitation Granda and Oliveros [54] proposed a new infrared cut-off for HDE, which is proportional to the square of the Hubble parameter squared $H^{2}$ and to the time derivative of the Hubble parameter $\dot{H}$, and dubbed this model the new HDE (NHDE) model. The energy density of the NHDE model is given by [54]

$\rho_{D}=3 M_{p}^{2}\left(\alpha H^{2}+\beta \dot{H}\right)$,

where $\alpha$ and $\beta$ are two positive constants. This model could avoid the problem of causality and could solve the coincidence problem [54]. In a more recent work, Li et al. [55] confirmed through the action principle that the NHDE model overcomes the causality and circular problems in the original HDE model and, putting constraints on the model from the Union2.1+BAO $+\mathrm{CMB}+\mathrm{H}_{0}$ data [55], got the goodness of-fit $\chi_{\min }^{2}=548.798$, which they found comparable with the results of the original HDE model (549.461) and the concordant $\Lambda \mathrm{CDM}$ model (550.354), and this led them to conclude that NHDE fit well to the data. Viewing scalar-field dark-energy models as an effective description of the underlying theory of dark energy, and considering the holographic vacuum energy scenario as pointing in the same direction, Granda and Oliveros [56] demonstrated how the scalar-field models can be used to describe the holographic energy density as effective theories; for this purpose they studied the correspondence between the quintessence, tachyon, K-essence, and dilaton energy densities with this NHDE in a flat FRW universe. Connecting these scalar-field models with NHDE, they found the explicit forms of the scalar fields and of the potentials in this reconstruction approach. Karami and Fehri [52] extended the work of [56] to non-flat FRW universe i.e. they studied a correspondence between NHDE and quintessence, tachyon, K-essence and dilaton scalar-field models in the presence of a spatial curvature and reconstructed the scalar field and potential. Sharif and Jawad [57] established the correspondence between the NHDE model and the quintessence, the tachyon, the K-essence, and the dilaton scalar-field models. In a recent work, Jawad et al. [58] explored holographic reconstruction of modified $f(R)$ Horava-Lifshitz gravity via a power-law scale factor and discussed the EoS parameter as well as stability of the reconstructed model and considered the quintessence era in the near future with an instability. The dependency of the evolution of equation of state, deceleration parameter, and cosmological evolution of the Hubble parameter on the parameters of the NHDE model were studied in Malekjiani et al. [59]. Considering the interaction between dark matter and NHDE, Debnath and Chattopadhyay [60] investigated the statefinder and the $\mathrm{Om}$ diagnostics and also checked the validity of the GSL of thermodynamics with the apparent horizon as the enveloping horizon of the universe.

Before demonstrating the current contribution in view of the work mentioned, let us have a brief overview of the theories of modified gravity as the current contribution is going to explore a cosmological reconstruction in the framework of a modified gravity theory. Modified gravity has become an essential part of theoretical cosmology nowadays [10,61,62]. It is proposed as a generalization of general relativity with the purpose to understand the qualitative change of gravitational interaction in the very early and/or very late universe. In particular, it is accepted nowadays that modified gravity may not only describe the early-time inflation and late-time acceleration but also may propose the unified consistent description of the universe evolution epochs sequence: inflation, radiation/matter dominance and dark energy [63]. Nojiri and Odintsov [62] summarized the usefulness of modified gravity as follows:

1. it provides us with a natural gravitational alternative for dark energy,

2. it presents a very natural unification of the early-time inflation and late-time acceleration thanks to the different 
role of gravitational terms relevant at small and at large curvature,

3. it may serve as the basis for a unified explanation of dark energy and dark matter.

Modified gravity models include $f(R)$ gravity (where $R$ is the Ricci scalar curvature) [64-66], $f(T)$ gravity (where $T$ represents the torsion scalar) $[67,68]$, scalar-tensor theories [69,70], braneworld models [71], Galileon gravity [72], Gauss-Bonnet gravity [73] and so on. Bamba et al. [74] investigated the future evolution of the dark-energy universe in modified gravities including $f(R)$ gravity, string-inspired scalar-Gauss-Bonnet and modified Gauss-Bonnet ones, and an ideal fluid with inhomogeneous equation of state, and they constructed several examples of the modified gravity that produces accelerating cosmologies ending at the finitetime future singularity by applying the reconstruction program. Cosmological evolution of the equation of state for dark energy $w_{\mathrm{DE}}$ in the exponential and logarithmic as well as their combination in the framework of $f(T)$ theories was studied in Bamba et al. [68]. A reconstruction scheme for modified gravity realizing a crossing of the phantom divide was proposed in Bamba et al. [75]. The appearance of finitetime future singularities in $f(T)$ gravity was demonstrated in Bamba et al. [76]. In another work, Bamba et al. [77] explored the cosmological evolution in a modified gravity $f(R)=R+c_{1}\left(1-e^{-c_{2} R}\right)$ and demonstrated that the latetime cosmic acceleration following the matter-dominated stage can be realized in that model. Bamba [78] showed that the crossing of the phantom divide can be realized in the combined $f(T)$ theory constructed with exponential and logarithmic terms.

Recently, various scalar-tensor theories have been considered extensively and one important example of the scalartensor theories is the Brans-Dicke (BD) theory of gravity, which was introduced by Brans and Dicke [79] to incorporate the Mach principle in Einstein's theory of gravity. BD theory is proposed as the natural extension of the KaluzaKlein idea of unification [80]. The BD parameter has some interesting properties as a candidate of DE when it has been studied in the non-minimally coupled regime [80-85]. The popularity of BD modified gravity $[86,87]$ lies in the fact that it naturally arises as the low energy limit of many other quantum gravity theories, like the Kaluza-Klein one or superstring theory. In this paper, we decided to consider the NHDE model in the framework of the chameleon Brans-Dicke modified gravity theory, in which there is a non-minimal coupling between the matter field and the scalar field $\phi$, which is usually known in literature by the name of 'chameleon field' [88,89], since its main physical properties strongly depend on the environment. Waterhouse [90] derived that the deviations from Newtonian gravity due to the chameleon field of the Earth are suppressed by nine orders of magnitude by the thin-shell effect. In a recent work, Chattopadhyay [91] studied the matter-chameleon coupling considering an extended holographic Ricci Dark Energy model in chameleon BransDicke cosmology. Instead, Bisbar [92] considered a generalized Brans-Dicke model, in which the scalar field has a potential function and it can couple non-minimally with the matter sector. Late-time dynamics of a chameleonic generalized Brans-Dicke cosmology with a power-law chameleonic function has been well studied in a recent paper of El-Nabulsi [93]. Other important works on chameleon gravity have been presented in [94-99].

The present contribution is organized as follows: in Sect. 2, we study the main cosmological properties of the NHDE model in the framework of Brans-Dicke chameleon cosmology. In Sect. 3, we make a correspondence between the reconstructed NHDE model and three different scalar-field models, i.e. the quintessence, the DBI-essence and the tachyon scalarfield models. Finally, in Sect. 4, we present the conclusions of this paper.

\section{NHDE MODEL in chameleon BD cosmology}

We begin this section with the description of the main cosmological properties of the chameleon Brans-Dicke (BD) theory.

According to BD theory, the scalar field is coupled nonminimally to the matter field via the action $S$ given by [92]

$$
\begin{aligned}
S= & \frac{1}{2} \int \mathrm{d}^{4} x \sqrt{-g} \\
& \times\left(\phi R-\frac{\omega}{\phi} g^{\mu \nu} \nabla_{\mu} \phi \nabla_{\nu} \phi-2 V+2 f(\phi) L_{m}\right)
\end{aligned}
$$

where $R$ indicates the Ricci scalar curvature, $\phi$ represents the Brans-Dicke scalar field with an associate potential $V(\phi)$, $\omega$ indicates the dimensionless Brans-Dicke parameter, $g^{\mu \nu}$ represents the metric tensor with determinant given by $g, L_{m}$ represents the matter Lagrangian, and, finally, $f(\phi)$ represents an arbitrary function of the scalar field $\phi$. The last term in the action $S$ given in Eq. (2) represents the term which gives us information as regards the interaction between the matter Lagrangian and the arbitrary function $f(\phi)$. We must also emphasize here that, in the limit case corresponding to $f(\phi)=1$, we obtain the standard BD cosmology.

Varying the action $S$ given in Eq. (2) with respect to the metric tensor $g_{\mu \nu}$ and $\phi$, we obtain the following field equations:

$\phi G_{\mu \nu}=T_{\mu \nu}^{\phi}+f(\phi) T_{\mu \nu}^{m}$,

$(2 \omega+3) \square \phi+2\left(2 V-V^{\prime} \phi\right)=T^{m} f-2 f^{\prime} \phi_{m}$,

where $G_{\mu \nu}$ is the Einstein tensor, $\square=\nabla^{\mu} \nabla_{\mu}$ (with $\nabla_{\mu}$ representing the covariant derivative) $T^{m}=g^{\mu \nu} T_{\mu \nu}^{m}$ and the prime denoting differentiation with respect to $\phi$. In Eq. (3), we have 


$$
\begin{aligned}
T_{\mu \nu}^{\phi}= & \frac{\omega}{\phi}\left(\nabla_{\mu} \phi \nabla_{\nu} \phi-\frac{1}{2} g_{\mu \nu} \nabla_{\alpha} \phi \nabla^{\alpha} \phi\right) \\
& +\left(\nabla_{\mu} \nabla_{\nu} \phi-g_{\mu \nu} \square \phi\right)-V(\phi) g_{\mu \nu}
\end{aligned}
$$

and

$T_{\mu \nu}^{m}=\frac{-2}{\sqrt{-g}} \frac{\delta\left(\sqrt{-g} L_{m}\right)}{\delta g^{\mu \nu}}$

Because of the explicit coupling between matter system and $\phi$, the stress tensor $T_{\mu \nu}^{m}$ is not divergence free. We now apply the above framework to a homogeneous and isotropic universe described by the Friedman-Robertson-Walker metric given by

$\mathrm{d} s^{2}=-\mathrm{d} t^{2}+a^{2}(t)\left(\frac{\mathrm{d} r^{2}}{1-k r^{2}}+r^{2} \mathrm{~d} \Omega^{2}\right)$.

The universe is open, closed or flat according to $k=-1$, +1 or 0 . Moreover, we find that $a(t)$ represents the scale factor (which gives information as regards the expansion of the universe), $r$ gives the radial component of the metric, $t$ indicates the cosmic time and $\mathrm{d} \Omega^{2}=r^{2}\left(\mathrm{~d} \theta^{2}+\sin ^{2} \theta \mathrm{d} \varphi^{2}\right)$ denotes the solid angle element (squared). $\theta$ and $\varphi$ are the usual azimuthal and polar angles, with $0 \leq \theta \leq \pi$ and $0 \leq \varphi \leq 2 \pi$. The coordinates $(r, t, \theta, \varphi)$ are known as comoving coordinates.

In a spatially flat universe (i.e. for $k=0$ ), Eqs. (3) and (6) yield [92]

$$
\begin{aligned}
& 3 H^{2}=\frac{f}{\phi} \rho+\frac{\omega}{2} \frac{\dot{\phi}^{2}}{\phi^{2}}-3 H \frac{\dot{\phi}}{\phi}+\frac{V}{\phi}, \\
& 3\left(\dot{H}+H^{2}\right)=-\frac{3 \rho}{\phi(2 \omega+3)} \\
& \quad \times\left\{\gamma \phi f^{\prime}+\left[\omega\left(\gamma+\frac{1}{3}\right)+1\right] f\right\} \\
& -\omega \frac{\dot{\phi}^{2}}{\phi^{2}}+3 H \frac{\dot{\phi}}{\phi}+\frac{1}{2 \omega+3}\left[3 V^{\prime}+(2 \omega-3) \frac{V}{\phi}\right], \\
& (2 \omega+3)(\ddot{\phi}+3 H \dot{\phi})-2\left(2 V-\phi V^{\prime}\right) \\
& =\rho\left[(1-3 \gamma) f+2 \gamma \phi f^{\prime}\right] .
\end{aligned}
$$

We must underline here that a dot indicates a time derivative while the prime indicates a derivative with respect to $\phi$.

In this paper, our purpose is to generalize the work of Karami and Fehri [52] to the NHDE model with energy density $\rho_{D}$ given by [51]

$\rho_{D}=\frac{3 \phi^{2}}{4 \omega}\left(\mu H^{2}+v \dot{H}\right)$

where $\mu$ and $v$ are two constant parameters and the overdot represents the first time derivative.
We consider the following ansatz for $\phi, V$, and $f[51,92]$ :

$$
\begin{aligned}
& \phi=\phi_{0} a^{\alpha}, \\
& V=V_{0} \phi^{\beta}, \\
& f=f_{0} \phi^{\gamma},
\end{aligned}
$$

where $\alpha, \beta$ and $\gamma$ are constant parameters and $\phi_{0}, V_{0}$ and $f_{0}$ are positive quantities representing the present day values of the corresponding quantities. One feature of taking this kind of ansatz is the scale invariance of the power laws. Given a relation in power-law form, scaling the argument by a constant factor $c$ causes only a proportional scaling of the function itself. Issues related to the power-law choice of the potential are discussed in [100,101].

Using the aforesaid ansatz in Eq. (8) we get the following differential equation for $H^{2}$ :

$$
\begin{aligned}
& \frac{\mathrm{d} H^{2}}{\mathrm{~d} a}+\left[\frac{2 \mu}{v}-\frac{8 e^{\alpha x} \phi_{0}\left(e^{\alpha x} \phi_{0}\right)^{-2-v} \omega}{3 f_{0} v}\right] H^{2} \\
& =-\frac{8\left(e^{\alpha x} \phi_{0}\right)^{-2+\beta-v} V_{0} \omega}{3 f_{0} v} .
\end{aligned}
$$

Solving Eq. (12), we get the expression of reconstructed $\tilde{H}^{2}$ as a function of the scale factor $a$ as follows:

$$
\begin{aligned}
\tilde{H}^{2}(a)= & \left(\frac{3}{8}\right)^{\eta_{4}} a^{\alpha(-2+\beta-v)} e^{-a^{s} \eta_{6}} \eta_{1} \eta_{3}\left(-a^{s} \eta_{5}\right)^{\eta_{4}} \\
& \times \Gamma\left[\eta_{2}, a^{s} \eta_{5}\right]
\end{aligned}
$$

where

$$
\begin{aligned}
& \eta_{1}=\frac{\left(\frac{3}{8}\right)^{-1+\frac{2 \mu}{s v}-\frac{\alpha(2-\beta+v)}{s}}}{f_{0} s v}, \\
& \eta_{2}=\frac{2 \mu+\alpha(-2+\beta-v) v}{s v} \\
& \eta_{3}=\phi_{0}^{-2+\beta-v} V_{0} \omega, \\
& \eta_{4}=-\frac{2 \mu}{s v}+\frac{\alpha(2-\beta+v)}{s} \\
& \eta_{5}=-\frac{8 \phi_{0}^{-1-v} \omega}{3 f_{0} \alpha v(1+v)} \\
& \eta_{6}=-\frac{8\left(a^{\alpha} \phi_{0}\right)^{-v} \omega}{3 f_{0} \phi_{0} \alpha v(1+v)}, \\
& s=-\alpha(1+v) .
\end{aligned}
$$

Moreover, we find that in Eq. (13) $\Gamma$ represents the Gamma function.

Subsequently, using the relation $\dot{H}=\frac{a}{2} \frac{\mathrm{d} H^{2}}{\mathrm{~d} a}$, we obtain the following relation for $\dot{\tilde{H}}(a)$ :

$$
\begin{aligned}
& \dot{\tilde{H}}(a)=-2^{-1-3 \eta_{4}} 3^{\eta_{4}} a^{\alpha(-2+\beta-v)} e^{-a^{s}\left(\eta_{5}+\eta_{6}\right)} \eta_{1} \eta_{3} \\
& \quad \times\left(-a^{s} \eta_{5}\right)^{\eta_{4}}\left(s\left(a^{s} \eta_{5}\right)^{\eta_{2}}-e^{a^{s} \eta_{5}}\left(s\left(\eta_{4}-a^{s} \eta_{6}\right)\right.\right. \\
& \left.\quad+\alpha(-2+\beta-v)) \Gamma\left[\eta_{2}, a^{s} \eta_{5}\right]\right) .
\end{aligned}
$$


Table 1 Values of reconstructed $w_{D}$ (Eq. (24)) for different choices of parameter values

\begin{tabular}{llll}
\hline Choice of $\alpha, \beta$ and $\gamma$ & $\begin{array}{l}\text { Vales of } w_{D} \\
(\mu=0.55, v=0.15)\end{array}$ & $\begin{array}{l}\text { Values of } w_{D} \\
(\mu=0.65, v=0.20)\end{array}$ & $\begin{array}{l}\text { Values of } w_{D} \\
(\mu=0.60, v=0.25)\end{array}$ \\
\hline$(\alpha=5, \beta=-0.7, \gamma=-0.9)$ & -1.47265 & -1.47040 & -1.47797 \\
$(\alpha=4.5, \beta=-0.6, \gamma=-1)$ & -1.57145 & -1.56864 & -1.57484 \\
$(\alpha=4.1, \beta=-0.8, \gamma=-1.1)$ & -1.25035 & -1.24863 & -1.25514 \\
\hline
\end{tabular}

We can use Eqs. (13) and (21) in Eq. (11) to reconstruct the density of the NHDE in chameleon BD cosmology, obtaining

$$
\begin{aligned}
& \rho_{D}(a)=-\frac{2^{-3-3 \eta_{4}} 3^{1+\eta_{4}}}{\omega}\left[a^{\alpha(\beta-\nu)} e^{-a^{s}\left(\eta_{5}+\eta_{6}\right)} \phi_{0}^{2} \eta_{1} \eta_{3}\right. \\
& \quad \times\left(-a^{s} \eta_{5}\right)^{\eta_{4}}\left\{s\left(a^{s} \eta_{5}\right)^{\eta_{2}} v-e^{a^{s} \eta_{5}}\left(2 \mu-v\left(-s \eta_{4}\right.\right.\right. \\
& \left.\left.\left.\left.+a^{s} s \eta_{6}+\alpha(2-\beta+v)\right)\right) \Gamma\left[\eta_{2}, a^{s} \eta_{5}\right]\right\}\right] .
\end{aligned}
$$

Since we are assuming that the universe is filled with NHDE, the conservation equation for $\rho_{D}$ is given by the following relation:

$\dot{\rho}_{D}+3 H \rho_{D}\left(1+w_{D}\right)=0$,

where $w_{D}$ represents the EoS parameter of DE.

From Eqs. (22) and (23), we derive the reconstructed equation of state (EoS) parameter $w_{D}$ as a function of the scale factor $a$ as follows:

$$
\begin{aligned}
& w_{D}(a)=-1-\left\{s ( a ^ { s } \eta _ { 5 } ) ^ { \eta _ { 2 } } \left(-2 \mu+v\left(-s\left(\eta_{2}+2 \eta_{4}\right.\right.\right.\right. \\
& \left.\left.\left.-a^{s}\left(\eta_{5}+2 \eta_{6}\right)\right)+2 \alpha(1-\beta+v)\right)\right)+e^{a^{s} \eta_{5}}\left(a^{2 s} s^{2} \eta_{6}^{2} v\right. \\
& +\left(s \eta_{4}+\alpha(\beta-v)\right)\left(2 \mu+\left(s \eta_{4}+\alpha(-2+\beta-v)\right) v\right) \\
& \left.-a^{s} s \eta_{6}\left(2 \mu+\left(s+2 s \eta_{4}+2 \alpha(-1+\beta-v)\right) v\right)\right) \\
& \left.\quad \times \Gamma\left[\eta_{2}, a^{s} \eta_{5}\right]\right\} \\
& \quad \times\left\{3 \left(s\left(a^{s} \eta_{5}\right)^{\eta_{2}} v-e^{a^{s} \eta_{5}}\left(2 \mu-v\left(-s \eta_{4}+a^{s} s \eta_{6}\right.\right.\right.\right. \\
& \left.\left.+\alpha(2-\beta+v))) \Gamma\left[\eta_{2}, a^{s} \eta_{5}\right]\right)\right\}^{-1} .
\end{aligned}
$$

We now plot the reconstructed cosmological parameters against the redshift $z$. In all figures, red, green, and blue lines correspond to $\{\mu=0.65, v=0.20\},\{\mu=0.60, v=0.25\}$, and $\{\mu=0.55, v=0.15\}$, respectively. For all other figures, the other parameters present in the equations we derived are set to $\alpha=5, \beta=-0.7, \gamma=-0.9, \phi_{0}=0.12, \omega=$ $-\frac{3}{2}+10^{-22}, f_{0}=1, V_{0}=2$. The choice of the value of the BD parameter $\omega$ is based on Ref. [102]. Observational results coming from SNeIa data suggest a range of possible values for the EoS parameter of $-1.67<w<-0.62$ [103]. Using a set of variations in the values of $\mu$ and $v$ in Eq. (24) we find that the results are in good agreement with [103]. In Table 1, we show a set of values of the reconstructed EoS based on the chosen values of the parameters. It is apparent from this table that the EoS parameter obeys $-1.67<w<-0.62$. In Fig. 1, we observe the evolution of the reconstructed Hubble parameter $\tilde{H}$ with redshift $z=a^{-1}-1$. We observe a

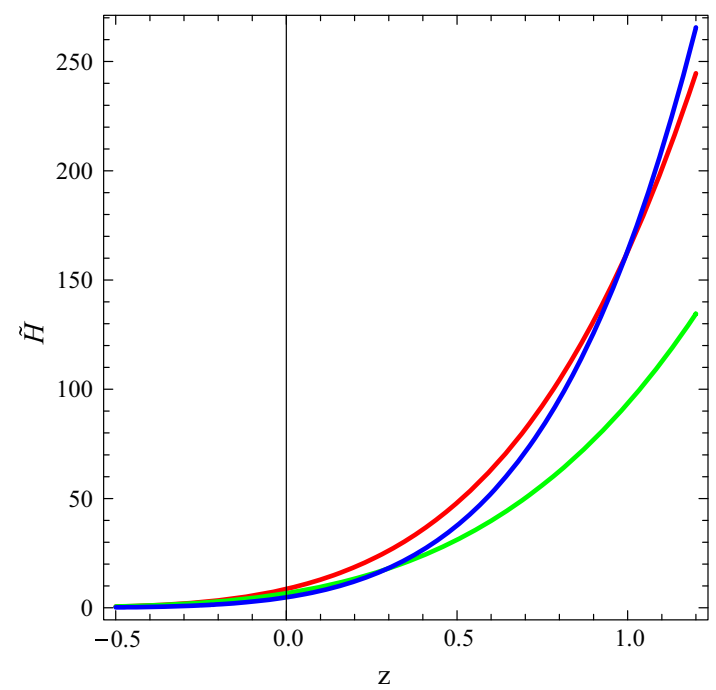

Fig. 1 Plot of the reconstructed Hubble parameter $\tilde{H}$ obtained in Eq. (13)

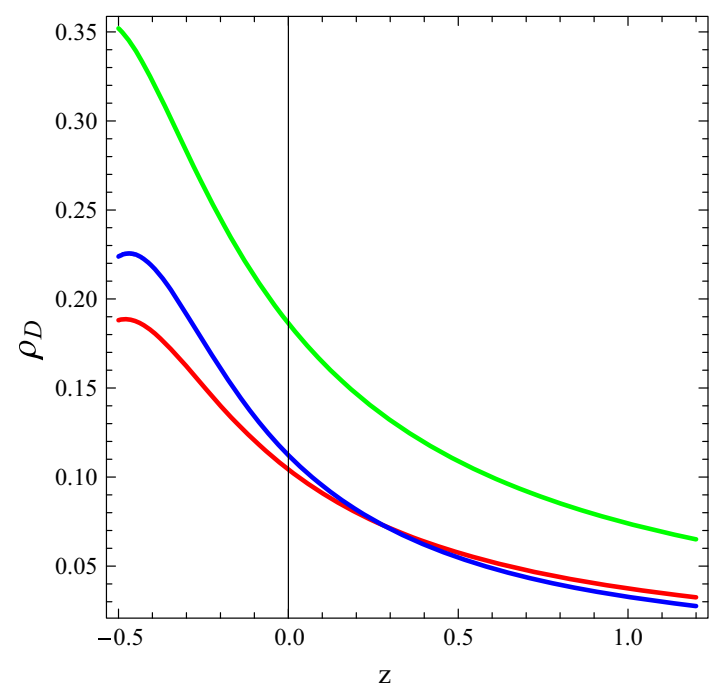

Fig. 2 Plot of the reconstructed energy density $\rho_{D}$. See Eq. (22)

decaying pattern of $\tilde{H}$ with evolution of the universe. This in consistent with the accelerated expansion of the universe. In Fig. 2, the reconstructed NHDE density is plotted and indicates its dominance with the evolution of the universe. This is consistent with the current dark-energy dominated era.

We now want to have a deeper look into Eq. (24), derived by the reconstructed $\tilde{H}$. In Eq. (24), the expression 


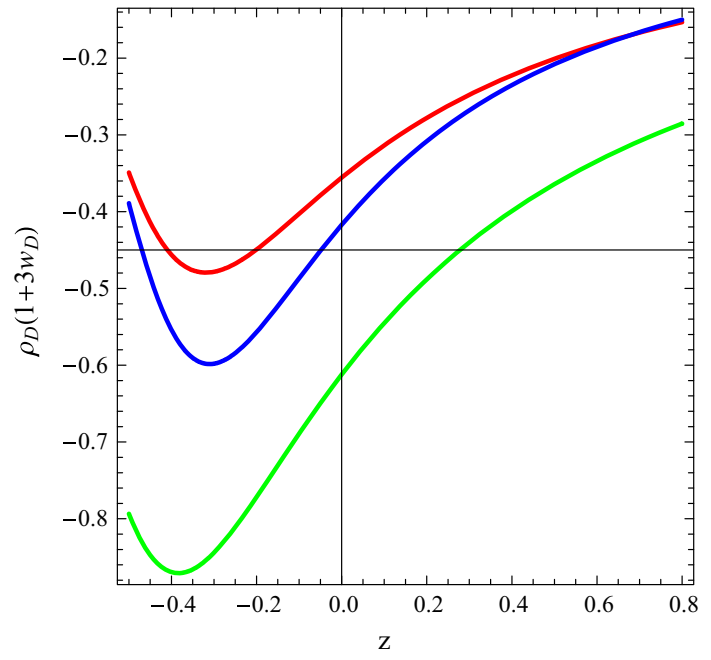

Fig. 3 Plot of the evolution of $\rho_{D}\left(1+3 w_{D}\right)$ (strong energy condition test). See Eqs. (22) and (24)

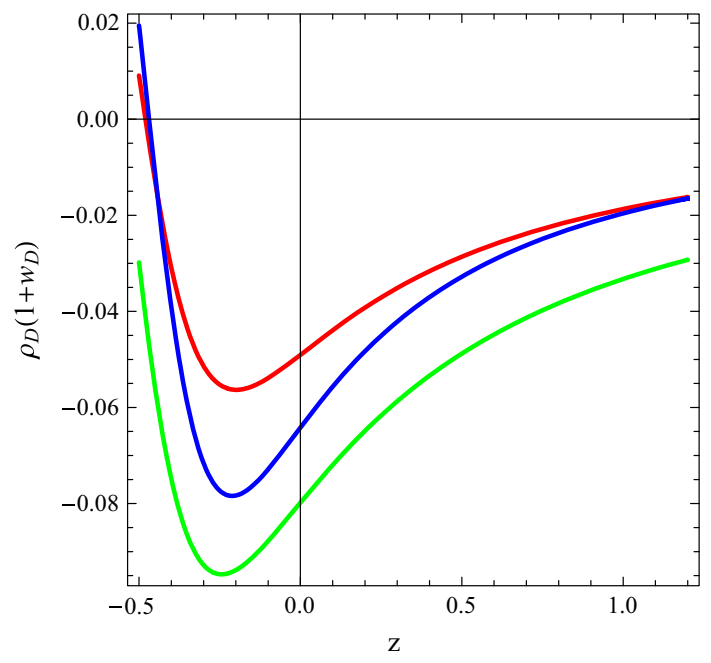

Fig. 4 Plot of the evolution of $\rho_{D}\left(1+w_{D}\right)$ (null energy condition test). See Eqs. (22) and (24)

$3\left(s\left(a^{s} \eta_{5}\right)^{\eta_{2}} v-e^{a^{s} \eta_{5}}\left(2 \mu-v\left(-s \eta_{4}+a^{s} s \eta_{6}+\alpha(2-\beta\right.\right.\right.$ $\left.+v))) \Gamma\left[\eta_{2}, a^{s} \eta_{5}\right]\right) \quad \neq 0$. Considering the forms of $\eta_{X}(X=1,2, \ldots, 6)$ it can be verified that the above expression is surely positive if $\alpha<0,-1<v<0$ or $\alpha>0, v<-1$. Since we have taken $\phi=\phi_{0} a^{\alpha}$, we have $\dot{\phi}=\alpha \phi H$. If $\alpha<0$, then the scalar field decays with the evolution of the universe.

Since the behavior of the remaining part of the expression is too complicated to make any inference on its "quintessence"- or "phantom"-like behavior, we only depend on the strong and null energy conditions to shed some light on the behavior of the equation of state parameter. The null energy condition is satisfied if $\rho_{D}\left(1+w_{D}\right) \geq 0$, while the strong energy condition is satisfied if $\rho_{D}\left(1+w_{D}\right) \geq$ 0 and $\rho_{D}\left(1+3 w_{D}\right) \geq 0$. Figures 3 and 4 indicate that both of the energy conditions are violated. This indicates a "phantom"-like behavior of the equation of state parameter.

In this section, we reconstructed the NHDE in the framework of chameleon BD cosmology. In the following section, our objective is to study the correspondence between this reconstructed NHDE model and the scalar-field models, namely (i) Quintessence dark energy, (ii) DBI-essence dark energy and (iii) Tachyon dark energy.

\subsection{Stability under a quantum correction}

Following [75,104] we examine the stability for the obtained solutions of the crossing of the phantom divide under a quantum correction of massless conformally invariant fields. Quantum effects produce the conformal anomaly [75]:

$T_{A}=b\left(F+\frac{2}{3} \square R\right)+b^{\prime} G+b^{\prime \prime} \square R$,

where

$F=\frac{1}{3} R^{2}-2 R_{i j} R^{i j}+R_{i j k l} R^{i j k l}$,

$G=R^{2}-4 R_{i j} R^{i j}+R_{i j k l} R^{i j k l}$.

In the FRW universe, we have

$F=0$,

$G=24\left(\dot{H} H^{2}+H^{4}\right)$.

For $N$ real scalar, $N_{1 / 2}$ Dirac spinor, $N_{1}$ vector fields, $N_{2}(=0$ or 1$)$ gravitons and $N_{\mathrm{HD}}$ higher derivative conformal scalars, we have the following expressions for $b$ and $b^{\prime}$ :

$b=\frac{N+6 N_{1 / 2}+12 N_{1}+611 N_{2}-8 N_{\mathrm{HD}}}{120(4 \pi)^{2}}$,

$b^{\prime}=-\frac{N+11 N_{1 / 2}+62 N_{1}+1411 N_{2}-28 N_{\mathrm{HD}}}{360(4 \pi)^{2}}$,

where $b^{\prime \prime}$ can be arbitrary. If we assume that $T_{A}$ can be given by the effective energy density $\rho_{A}$ and pressure $p_{A}$ from the conformal anomaly as

$\dot{\rho}_{A}+3 H\left(\rho_{A}+p_{A}\right)=0$,

the following expressions for $\rho_{A}$ and $p_{A}$ can be found:

$\rho_{A}=-\frac{1}{a^{4}} \int \mathrm{d} t a^{4} H T_{A}$,

$p_{A}=-\frac{1}{3 a^{4}} \int \mathrm{d} t a^{4} H T_{A}+\frac{T_{A}}{3}$.

At phantom crossing, we must have $\dot{H}=0$. If we assume that the magnitude of the Hubble rate $H$ could be the order of the present Hubble constant $H_{0}$, then for the phantom crossing we have 
$\rho_{D}=\frac{3 \phi^{2}}{4 \omega} \tilde{H}_{0}^{2} \mu$

where $\tilde{H}_{0}^{2}=\left.\tilde{H}^{2}\right|_{(a=1)}=\left(\frac{3}{8}\right)^{\eta_{4}} e^{-\eta_{6}} \eta_{1} \eta_{3}\left(-\eta_{5}\right)^{\eta_{4}} \Gamma\left[\eta_{2}, \eta_{5}\right]$.

The results obtained in Eqs. (33) and (34) tell us that we may assume $\rho_{A} \sim p_{A} \sim T_{A}$. Then we find [104]:

$\rho_{A} \sim p_{A} \sim C \tilde{H}_{0}^{4}$

where $C$ represents a dimensionless constant of the order of $\sim 10^{2 \sim 3}$. Thus, for the reconstructed model, we obtain

$\frac{\rho_{D}}{\rho_{A}}=\frac{3 \phi^{2} \mu}{4 C \omega \tilde{H}_{0}^{2}} \approx 10^{19} \frac{\mu}{\tilde{H}_{0}^{2}} ;$

hence we can conclude that

$\left|\rho_{D}\right| \gg\left|\rho_{A}\right|$.

Therefore, the quantum correction could be small when the phantom crossing occurs and the solutions of the phantom crossing obtained in this paper could be stable under the quantum correction.

\section{New holographic reconstruction of scalar-field models in $\mathrm{BD}$ cosmology}

Sahni and Starobinsky [17] discussed various aspects of reconstructing the expansion history of the Universe and to probe the nature of dark energy. Below, we will study the correspondence between NHDE model and the quintessence, the DBI-essence and the tachyon scalar-field models in the framework of a flat chameleon Brans-Dicke universe. We will also reconstruct the potentials and the dynamics for these scalar-field models. We can give the related results of scalar fields and potentials for the NHDE model in the flat chameleon Brans-Dicke universe. In order to establish this correspondence, we compare the energy density of the NHDE model given in Eq. (22) with the corresponding energy density of the scalar-field model, and we also equate the EoS for these scalar models with the EoS for the NHDE model given in Eq. (24). We must also emphasize here that we indicate the scalar field with $\varphi$ in order to make it different from the scalar field $\phi$ in Brans-Dicke theory.

\subsection{Reconstruction of quintessence dark-energy model}

Quintessence is a dynamical, evolving, spatially inhomogeneous component with negative pressure. Unlike a cosmological constant, the quintessential pressure and energy density evolve with time, and the EoS parameter may also do so. A common model of quintessence is the energy density associated with a scalar field $Q$ slowly rolling down a potential $V(Q)$. A detailed discussion of quintessence dark energy is available in the review [105]. The energy density $\rho_{Q}$ and pressure $p_{Q}$ of the quintessence scalar field $\varphi$ are given, respectively, by [45-47]:

$\rho_{Q}=\frac{1}{2} \dot{\varphi}^{2}+V(\varphi)$,

$p_{Q}=\frac{1}{2} \dot{\varphi}^{2}-V(\varphi)$.

Moreover, the EoS parameter can be written as follows:

$w_{Q}=\frac{p_{Q}}{\rho_{Q}}=\frac{\dot{\varphi}^{2}-2 V(\varphi)}{\dot{\varphi}^{2}+2 V(\varphi)}$.

As we are reconstructing the quintessence model based on NHDE in the framework of chameleon BD cosmology, we shall consider $\rho_{Q}=\rho_{D}$ and $w_{Q}=w_{D}$. Hence, we have

$$
\begin{aligned}
\dot{\varphi}^{2} & =\rho_{D}\left(1+w_{D}\right), \\
V(\varphi) & =\frac{\rho_{D}}{2}\left(1-w_{D}\right),
\end{aligned}
$$

where $\rho_{D}$ and $w_{D}$ are given in Eqs. (22) and (24), respectively. Based on the reconstructed Hubble parameter, we express $\dot{\varphi}^{2}$ and $V(\varphi)$ as functions of the scale factor $a$ as follows:

$$
\begin{aligned}
\dot{\varphi} & (a)^{2}=-\frac{1}{\omega} 3^{\eta_{4}} 8^{-1-\eta_{4}} a^{\alpha(\beta-v)} e^{-a^{s}\left(\eta_{5}+\eta_{6}\right)} \phi_{0}^{2} \eta_{1} \eta_{3} \\
& \times\left(-a^{s} \eta_{5}\right)^{\eta_{4}}\left(s\left(a^{s} \eta_{5}\right)^{\eta_{2}}\right. \\
& \times\left(-2 \mu+v\left(-s\left(\eta_{2}+2 \eta_{4}-a^{s}\left(\eta_{5}+2 \eta_{6}\right)\right)\right.\right. \\
& +\alpha(2-2 \beta+2 v)))+e^{a^{s} \eta_{5}}\left(\alpha ^ { 2 } v \left(\beta^{2}-2 \beta(1+v)\right.\right. \\
& +v(2+v))+s\left(s \eta_{4}^{2} v+2 \eta_{4}\left(\mu-a^{s} s \eta_{6} v\right)\right. \\
& \left.+a^{s} \eta_{6}\left(-2 \mu+s\left(-1+a^{s} \eta_{6}\right) v\right)\right) \\
& +2 \alpha\left(\beta\left(\mu+s\left(\eta_{4}-a^{s} \eta_{6}\right) v\right)\right. \\
& \left.-v\left(\mu+s\left(\eta_{4}-a^{s} \eta_{6}\right)(1+v)\right) \Gamma\left[\eta_{2}, a^{s} \eta_{5}\right]\right), \\
& \\
& (\varphi(a))=-\frac{2^{-4-3 \eta_{4}} 3^{1+\eta_{4}}}{\omega} a^{\alpha(\beta-v)} e^{-a^{s}\left(\eta_{5}+\eta_{6}\right)} \phi_{0}^{2} \eta_{1} \eta_{3} \\
& \times\left(-a^{s} \eta_{5}\right)^{\eta_{4}}\left(s\left(a^{s} \eta_{5}\right)^{\eta_{2}} v\right. \\
& -e^{a^{s} \eta_{5}}\left(2 \mu-v\left(-s \eta_{4}+a^{s} s \eta_{6}+\alpha(2-\beta+v)\right)\right) \\
& \left.\times \Gamma\left[\eta_{2}, a^{s} \eta_{5}\right]\right)\left(1-\left(s\left(a^{s} \eta_{5}\right)^{\eta_{2}}\right.\right. \\
& \times\left(-2 \mu+v\left(-3-s\left(\eta_{2}+2 \eta_{4}-a^{s}\left(\eta_{5}+2 \eta_{6}\right)\right)\right.\right. \\
& +2 \alpha(1-\beta+v))) \\
& +e^{a^{s} \eta_{5}}\left(a^{2 s} s^{2} \eta_{6}^{2} v+\left(3+s \eta_{4}+\alpha(\beta-v)\right)\right. \\
& \times\left(2 \mu+\left(s \eta_{4}+\alpha(-2+\beta-v)\right) v\right) \\
& \left.-a^{s} s \eta_{6}\left(2 \mu+\left(3+s+2 s \eta_{4}+2 \alpha(-1+\beta-v)\right) v\right)\right) \\
& \left.\times \Gamma\left[\eta_{2}, a^{s} \eta_{5}\right]\right) /\left(3 \left(s\left(a^{s} \eta_{5}\right)^{\eta_{2}} v-e^{a^{s} \eta_{5}}(2 \mu\right.\right. \\
& \left.\left.\left.\left.-v\left(-s \eta_{4}+a^{s} s \eta_{6}+\alpha(2-\beta+v)\right)\right) \Gamma\left[\eta_{2}, a^{s} \eta_{5}\right]\right)\right)\right) .
\end{aligned}
$$


3.2 Reconstruction of DBI-essence dark-energy model

During the last few years, there have been many works aiming at connecting string theory with inflation, which is also a phase of accelerated expansion. Martin and Yamaguchi [106] introduced a scalar-field model of dark energy with a nonstandard Dirac-Born-Infeld (DBI) kinetic term. This model is dubbed "DBI-essence dark energy" and the energy density $\rho_{\text {DBI }}$ and the pressure $p_{\text {DBI }}$ of the DBI-essence model are given, respectively, by [106]

$$
\begin{aligned}
& \rho_{\mathrm{DBI}}=(\eta-1) T(\varphi)+V(\varphi), \\
& p_{\mathrm{DBI}}=\left(\frac{\eta-1}{\eta}\right) T(\varphi)-V(\varphi),
\end{aligned}
$$

where

$$
\eta=\frac{1}{\sqrt{1-\frac{\dot{\varphi}^{2}}{T}}} .
$$

The EoS parameter for the DBI-essence scalar-field model can be written as follows:

$w_{\mathrm{DBI}}=\frac{p_{\mathrm{DBI}}}{\rho_{\mathrm{DBI}}}=\frac{(\eta-1) T(\varphi)-V(\varphi) \eta}{\eta((\eta-1) T(\varphi)+V(\varphi))}$.

In the present work, we shall assume that $T=n \dot{\varphi}^{2}$, where $n>0$. Since we are considering the correspondence between DBI-essence dark energy and the reconstructed NHDE model, we consider $\rho_{\mathrm{DBI}}=\rho_{D}$ and $w_{\mathrm{DBI}}=w_{D}$. Based on Eq. (46) we get the reconstructed scalar field $\varphi$ as a function of the scale factor $a$ as follows:

$$
\begin{aligned}
\dot{\varphi} & (a)^{2}=-\frac{3^{\eta_{4}} 8^{-1-\eta_{4}} a^{\alpha(\beta-v)}}{\omega} \\
& \times e^{-a^{s}\left(\eta_{5}+\eta_{6}\right)} \sqrt{1-\frac{1}{n}} \phi_{0}^{2} \eta_{1} \eta_{3}\left(-a^{s} \eta_{5}\right)^{\eta_{4}}\left(s\left(a^{s} \eta_{5}\right)^{\eta_{2}}\right. \\
& \times\left(-2 \mu+v\left(-s\left(\eta_{2}+2 \eta_{4}-a^{s}\left(\eta_{5}+2 \eta_{6}\right)\right)\right.\right. \\
& +\alpha(2-2 \beta+2 v)))+e^{a^{s} \eta_{5}} \\
& \times\left(\alpha^{2} v\left(\beta^{2}-2 \beta(1+v)+v(2+v)\right)\right. \\
& +s\left(s \eta_{4}^{2} v+2 \eta_{4}\left(\mu-a^{s} s \eta_{6} v\right)\right. \\
& \left.+a^{s} \eta_{6}\left(-2 \mu+s\left(-1+a^{s} \eta_{6}\right) v\right)\right) \\
& +2 \alpha\left(\beta\left(\mu+s\left(\eta_{4}-a^{s} \eta_{6}\right) v\right)\right. \\
& \left.\left.\left.-v\left(\mu+s\left(\eta_{4}-a^{s} \eta_{6}\right)(1+v)\right)\right)\right) \Gamma\left[\eta_{2}, a^{s} \eta_{5}\right]\right)
\end{aligned}
$$

$$
\begin{aligned}
& V(\varphi(a))=\frac{3^{\eta_{4}} 8^{-1-\eta_{4}}}{\omega} a^{\alpha(\beta-v)} e^{-a^{s}\left(\eta_{5}+\eta_{6}\right)} \\
& \quad \times \phi_{0}^{2} \eta_{1} \eta_{3}\left(-a^{s} \eta_{5}\right)^{\eta_{4}}\left(-3\left(s\left(a^{s} \eta_{5}\right)^{\eta_{2}} v-e^{a^{s} \eta_{5}}\right.\right. \\
& \quad \times\left(2 \mu-v\left(-s \eta_{4}+a^{s} s \eta_{6}+\alpha(2-\beta+v)\right)\right) \\
& \left.\quad \times \Gamma\left[\eta_{2}, a^{s} \eta_{5}\right]\right)
\end{aligned}
$$

$$
\begin{aligned}
& +\left(-1+\sqrt{\frac{n}{n-1}}\right) \sqrt{\frac{n-1}{n}} n\left(s\left(a^{s} \eta_{5}\right)^{\eta_{2}}\right. \\
& \times\left(-2 \mu+v\left(-s\left(\eta_{2}+2 \eta_{4}-a^{s}\left(\eta_{5}+2 \eta_{6}\right)\right)\right.\right. \\
& +\alpha(2-2 \beta+2 v)))+e^{a^{s} \eta_{5}} \\
& \times\left(\alpha^{2} v\left(\beta^{2}-2 \beta(1+v)+v(2+v)\right)\right. \\
& +s\left(s \eta_{4}^{2} v+2 \eta_{4}\left(\mu-a^{s} s \eta_{6} v\right)\right. \\
& \left.+a^{s} \eta_{6}\left(-2 \mu+s\left(-1+a^{s} \eta_{6}\right) v\right)\right) \\
& +2 \alpha\left(\beta\left(\mu+s\left(\eta_{4}-a^{s} \eta_{6}\right) v\right)\right. \\
& \left.\left.\left.\left.-v\left(\mu+s\left(\eta_{4}-a^{s} \eta_{6}\right)(1+v)\right)\right)\right) \Gamma\left[\eta_{2}, a^{s} \eta_{5}\right]\right)\right) .
\end{aligned}
$$

\subsection{Reconstruction of tachyon dark-energy model}

A tachyonic condensate in a class of string theories can be described by an effective scalar field with a Lagrangian of the form $L=-V(\phi)\left(1-\partial_{a} \phi \partial^{a} \phi\right)^{1 / 2}$. Since this Lagrangian has also a potential function $V(\phi)$, any form of cosmological evolution (that is, any $a(t))$ can be obtained with the tachyonic field as the source by choosing $V(\phi)$ suitably [107]. The cosmological effects of homogeneous tachyon matter coexisting with non-relativistic matter and radiation have been studied by [108]. The energy density $\rho_{T}$ and the pressure $p_{T}$ of the tachyon scalar-field model are given, respectively, by [107]

$$
\begin{aligned}
\rho_{T} & =\frac{V(\varphi)}{\sqrt{1-\dot{\varphi}^{2}}}, \\
p_{T} & =-V(\varphi) \sqrt{1-\dot{\varphi}^{2}},
\end{aligned}
$$

while the EoS parameter can be written as follows:

$w_{T}=\frac{p_{T}}{\rho_{T}}=\dot{\varphi}^{2}-1$.

For the correspondence under consideration, we have $\rho_{T}=$ $\rho_{D}$ and $w_{D}=w_{T}$. Using the same procedure as used before, we reconstruct the scalar field $\varphi$ and the potential $V(\varphi)$ as follows:

$$
\begin{aligned}
& \dot{\varphi}(a)^{2}=1+\left(s ( a ^ { s } \eta _ { 5 } ) ^ { \eta _ { 2 } } \left(-2 \mu+v\left(-3-s\left(\eta_{2}\right.\right.\right.\right. \\
& \left.\left.\left.\quad+2 \eta_{4}-a^{s}\left(\eta_{5}+2 \eta_{6}\right)\right)+2 \alpha(1-\beta+v)\right)\right) \\
& \quad+e^{a^{s} \eta_{5}}\left(a^{2 s} s^{2} \eta_{6}^{2} v+\left(3+s \eta_{4}+\alpha(\beta-v)\right)\right. \\
& \quad \times\left(2 \mu+\left(s \eta_{4}+\alpha(-2+\beta-v)\right) v\right)-a^{s} s \eta_{6} \\
& \left.\quad \times\left(2 \mu+\left(3+s+2 s \eta_{4}+2 \alpha(-1+\beta-v)\right) v\right)\right) \\
& \left.\quad \times \Gamma\left[\eta_{2}, a^{s} \eta_{5}\right]\right) \times\left(3 \left(s\left(a^{s} \eta_{5}\right)^{\eta_{2}} v-e^{a^{s} \eta_{5}}\right.\right. \\
& \quad \times\left(2 \mu-v\left(-s \eta_{4}+a^{s} s \eta_{6}+\alpha(2-\beta+v)\right)\right) \\
& \left.\left.\quad \times \Gamma\left[\eta_{2}, a^{s} \eta_{5}\right]\right)\right)^{-1},
\end{aligned}
$$




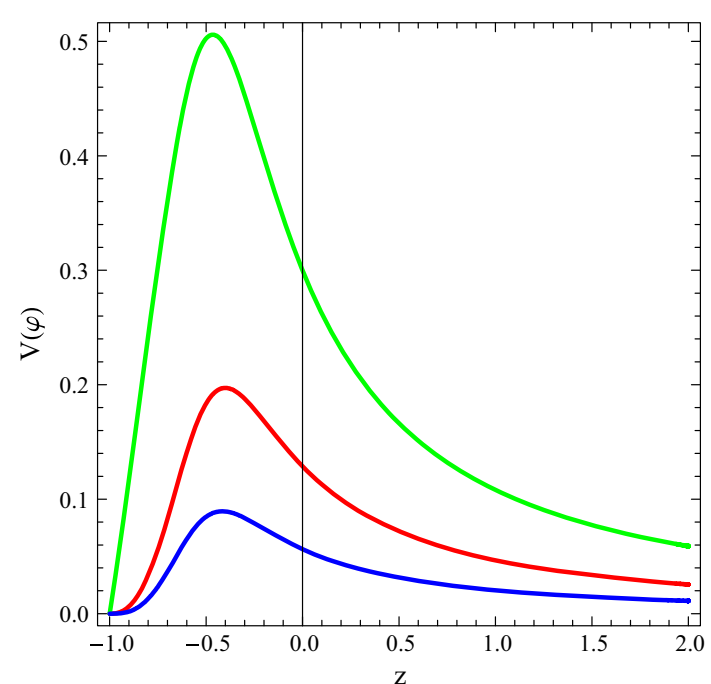

Fig. 5 Plot of the evolution of the reconstructed potential $V(\varphi)$ for the reconstructed quintessence dark energy model. See Eq. (45)

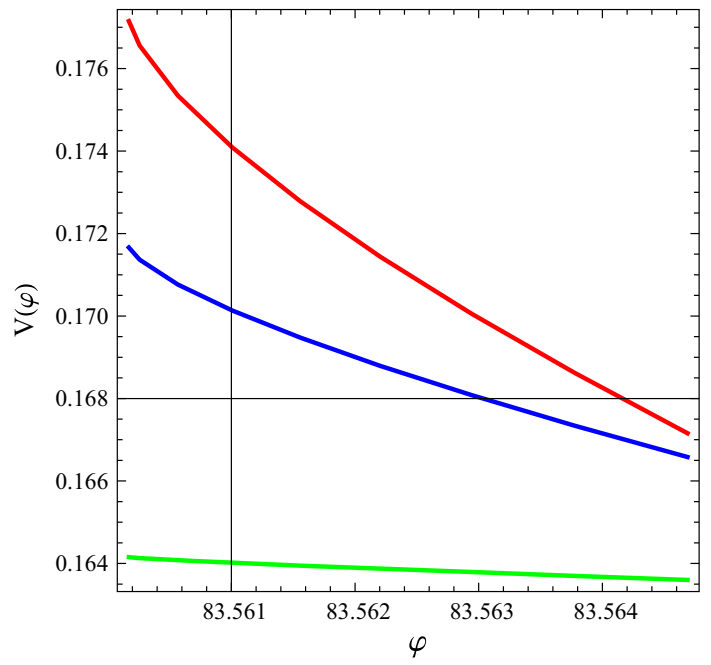

Fig. 6 Plot of the evolution of the reconstructed potential $V(\varphi)$ (Eq. (45)) with reconstructed scalar field $\varphi$ (Eq. (44)) of the quintessence dark energy model

$$
\begin{aligned}
& V(\varphi(a))=\left[-\frac{3^{1+2 \eta_{4}} a^{2 \alpha(\beta-v)} e^{-2 a^{s}\left(\eta_{5}+\eta_{6}\right)} \phi_{0}^{4} \eta_{1}^{2} \eta_{3}^{2}}{\omega^{2}}\right. \\
& \quad \times 2^{-6\left(1+\eta_{4}\right)}\left(-a^{s} \eta_{5}\right)^{2 \eta_{4}}\left(s\left(a^{s} \eta_{5}\right)^{\eta_{2}}\right. \\
& \times\left(-2 \mu+v\left(-3-s\left(\eta_{2}+2 \eta_{4}-a^{s}\left(\eta_{5}+2 \eta_{6}\right)\right)\right.\right. \\
& +2 \alpha(1-\beta+v)))+e^{a^{s} \eta_{5}}\left(a^{2 s} s^{2} \eta_{6}^{2} v\right. \\
& +\left(3+s \eta_{4}+\alpha(\beta-v)\right) \\
& \quad \times\left(2 \mu+\left(s \eta_{4}+\alpha(-2+\beta-v)\right) v\right) \\
& \left.-a^{s} s \eta_{6}\left(2 \mu+\left(3+s+2 s \eta_{4}+2 \alpha(-1+\beta-v)\right) v\right)\right) \Gamma \\
& \left.\times\left[\eta_{2}, a^{s} \eta_{5}\right]\right)\left(s\left(a^{s} \eta_{5}\right)^{\eta_{2}} v-e^{a^{s} \eta_{5}}\left(2 \mu-v\left(-s \eta_{4}+a^{s} s \eta_{6}\right.\right.\right. \\
& \left.\left.+\alpha(2-\beta+v)) \Gamma\left[\eta_{2}, a^{s} \eta_{5}\right]\right)\right]^{1 / 2} .
\end{aligned}
$$

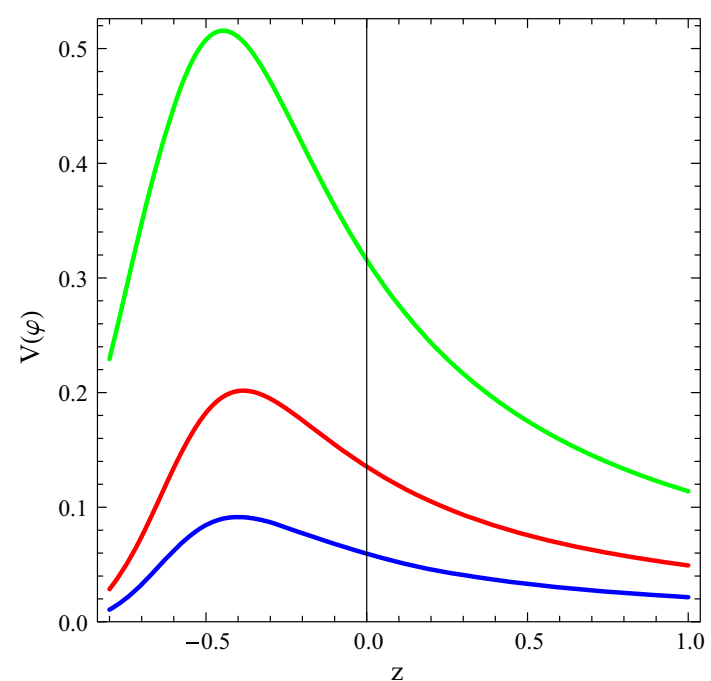

Fig. 7 Plot of the evolution of the reconstructed potential $V(\varphi)$ for the reconstructed DBI-essence dark-energy model. See Eq. (51). We have chosen $n=1.5$

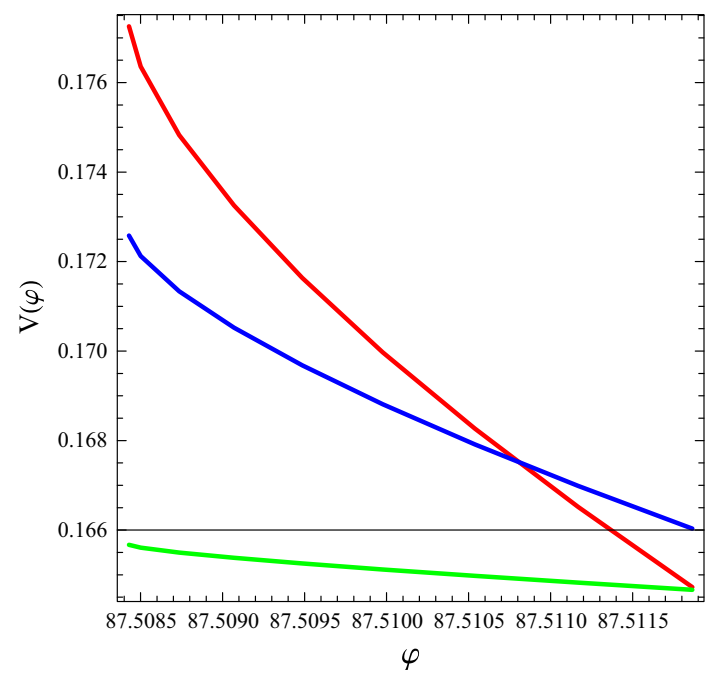

Fig. 8 Plot of the evolution of the reconstructed potential $V(\varphi)$ (Eq. (51)) with reconstructed scalar field $\varphi$ (Eq. (50)) of the DBI-essence dark-energy model. We have chosen $n=1.5$

\subsection{Discussion}

In this paper, we studied the main cosmological properties of the new holographic dark energy (HNDE) model in the framework of Brans-Dicke chameleon cosmology. We considered a particular ansatz for the parameters $\phi, V$, and $f$ in which their expressions are given in the power-law form. We decided to consider different aspects to study. First of all, we reconstructed the expression of the Hubble parameter $H$ and, accordingly, the expression of the density $\rho_{D}$ of the NHDE in the context of chameleon Brans-Dicke chameleon cosmology. We also tested the Weak Energy condition (WEC) and the Strong Energy Condition (SEC) for the 


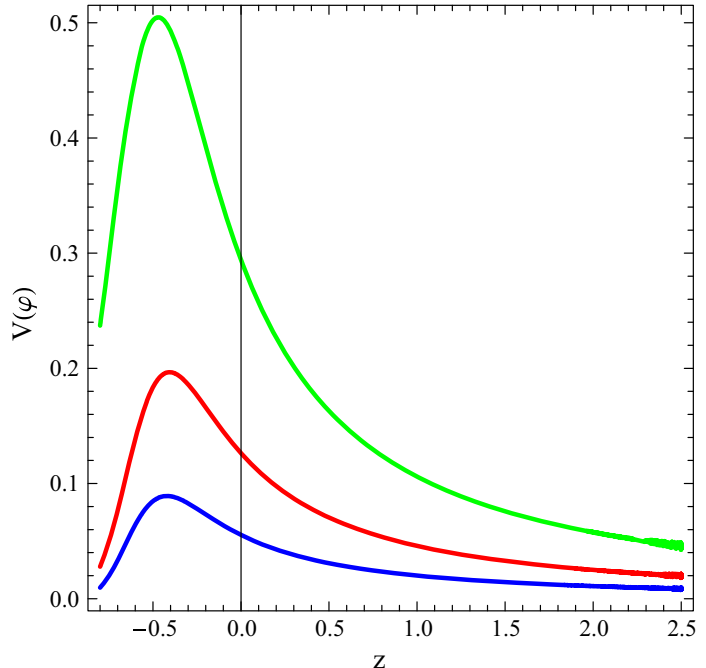

Fig. 9 Plot of the evolution of the reconstructed potential $V(\varphi)$ for the reconstructed tachyon dark-energy model. See Eq. (56)

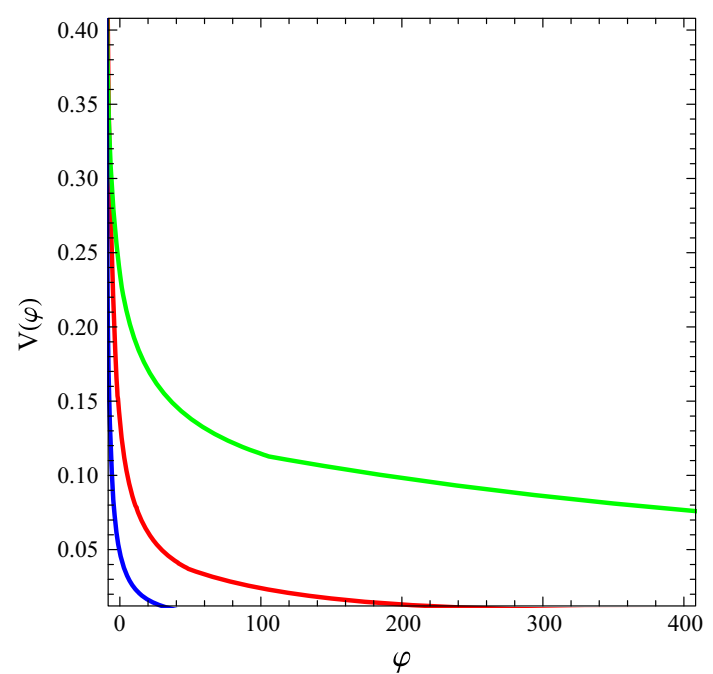

Fig. 10 Plot of the evolution of the reconstructed potential $V(\varphi)$ (Eq. (56)) with reconstructed scalar field $\varphi$ (Eq. (55)) of the tachyon dark-energy model

reconstructed model we obtained. Considering three cases, namely $\{\mu=0.65, v=0.20\},\{\mu=0.60, v=0.25\}$, and $\{\mu=0.55, v=0.15\}$ setting the other parameters to $\alpha=5, \beta=-0.7, \gamma=-0.9, \phi_{0}=0.12, \omega=$ $-\frac{3}{2}+10^{-22}, f_{0}=1, V_{0}=2$, and the BD parameter $\omega$ following [102] we have computed the reconstructed EoS parameter. Observational results coming from SNeIa data suggest a limit of the EoS parameter: $-1.67<w<-0.62$ [103]. Using the set values of $\mu$ and $v$ in Eq. (24) we found that the results are in good agreement with the observations of [103]. Finally, we reconstructed three scalar-field models of dark energy (namely, the quintessence, the DBI-essence, and the tachyon ones) based on the NHDE model in the framework of BD cosmology. For the three scalar-field mod-

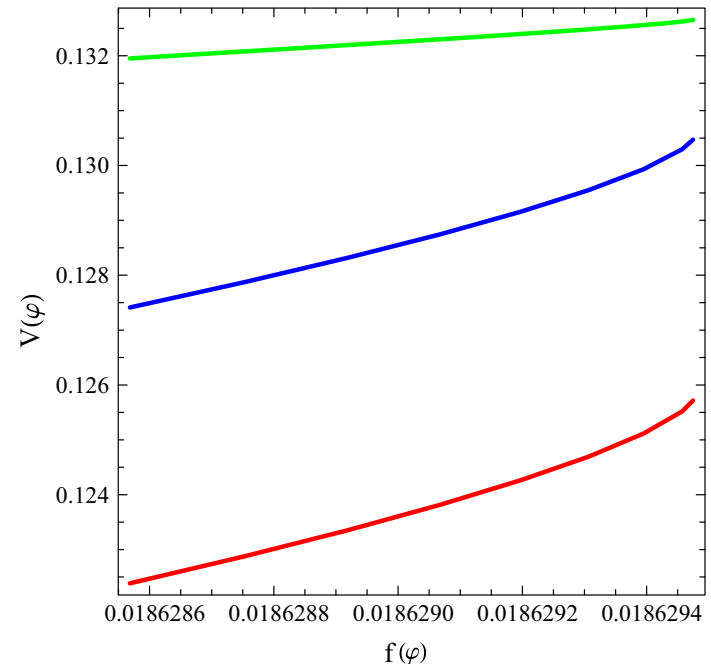

Fig. 11 Plot of the evolution of the reconstructed potential $V(\varphi)$ with $f(\varphi)$ for the quintessence dark energy model

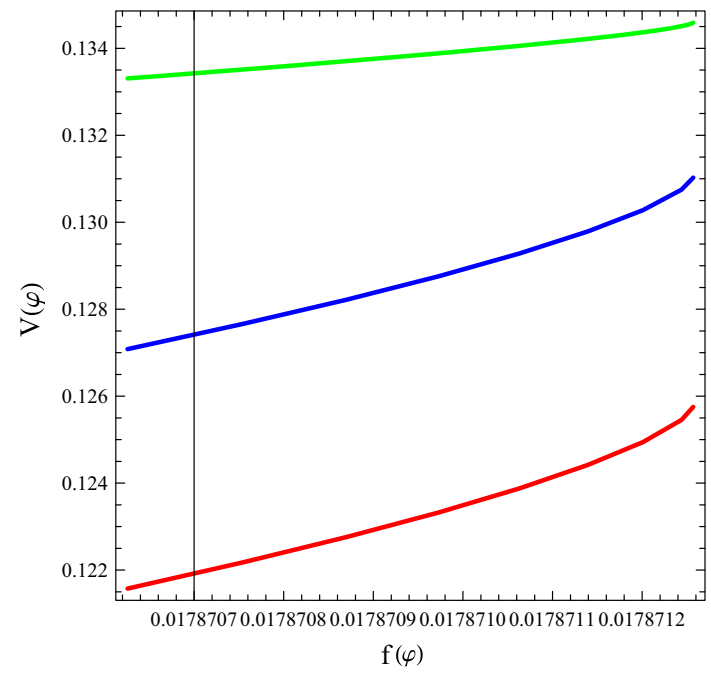

Fig. 12 Plot of the evolution of the reconstructed potential $V(\varphi)$ with $f(\varphi)$ for the DBI-essence dark-energy model

els we considered, we have reconstructed the corresponding potentials and scalar fields. To further elucidate our reconstructions, we have plotted the reconstructed potential $V(\varphi)$ against $z$ and made parametric plots between $\varphi$ and $V(\varphi)$ in Figs. 5, 6, 7, 8, 9, and 10. It is apparent from the plots that the potential $V(\varphi)$ is increasing up to redshifts of the order of $z \approx-0.5$; afterwards it starts to decay. In the plots of $\varphi-$ $V(\varphi)$, it appears that the potential has a decreasing behavior with the scalar field $\varphi$.

In order to have a look into the behavior of the reconstructed potential against coupling function $f$ we discuss Figs. 11, 12, and 13, where we observe that the reconstructed potentials are increasing with $f$. This indicates that the potential increases as the matter-chameleon coupling is getting stronger. It is further noted that the rate of increase in the 


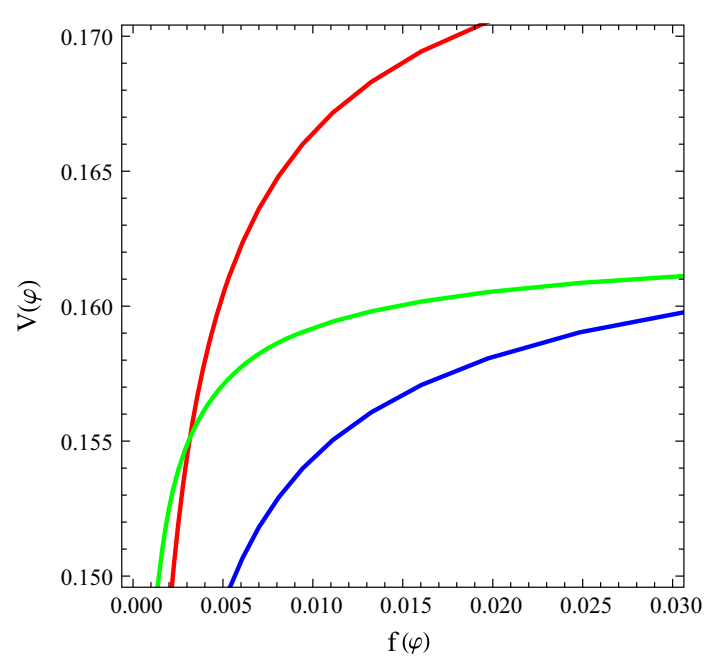

Fig. 13 Plot of the evolution of the reconstructed potential $V(\varphi)$ with $f(\varphi)$ for the tachyon dark-energy model

potential is much higher in the case of the tachyon than the cases of quintessence and DBI.

\section{Concluding remarks}

In the present work we have used a reconstruction scheme for a new holographic dark-energy model with energy density given by $\rho_{D}=\frac{3 \phi^{2}}{4 \omega}\left(\mu H^{2}+\nu \dot{H}\right)$ in the framework of Brans-Dicke cosmology taking the ansatz $\phi=\phi_{0} a^{\alpha}, V=$ $V_{0} \phi^{\beta}, f=f_{0} \phi^{\gamma}$. The results of the study are:

- Considering $\rho=\rho_{D}$ in the first modified field equation of $\mathrm{BD}$ theory leads to a linear differential equation which could be solved analytically to obtain a solution for the reconstructed Hubble parameter in terms of scale factor $a$; when plotted against the redshift $z=a^{-1}-1$, it exhibited decaying pattern with the evolution of the universe (i.e., decreases in $z$ ) and this is consistent with the accelerated expansion of the universe.

- The NHDE energy density, as reconstructed through Hubble parameter, when plotted against $z$, is found to increase with the evolution of the universe and it is consistent with the evolution of the universe from matter to dark-energy domination.

- The violation of the strong energy condition, as expected in the framework of Einstein gravity, has also been found for the reconstructed NHDE model in the framework of BD gravity.

- The reconstructed equation of state (EoS) parameter has been found to exhibit a "phantom"-like behavior, i.e. $w_{D}<-1$.

- Considering three different combinations of the parameters $\mu$ and $\nu$, namely $\{\mu=0.65, \nu=0.20\},\{\mu=$
$0.60, v=0.25\}$ and $\{\mu=0.55, v=0.15\}$ and setting the other parameters as $\alpha=5, \beta=-0.7, \gamma=$ $-0.9, \phi_{0}=0.12, \omega=-\frac{3}{2}+10^{-22}, f_{0}=1, V_{0}=2$ and the BD parameter $\omega$ following [102], we have computed the reconstructed EoS parameter for the reconstructed NHDE. The observational results coming from the SNeIa data suggest a limit of the EoS parameter: $-1.67<w<-0.62$ [103]. Using a set of variations in the values of $\mu$ and $v$ in Eq. (24) we found that the results are in good agreement with observations of [103] (see Table 1).

In the following phase of the study, we considered the correspondence between the reconstructed new holographic dark energy in the framework of BD gravity and some scalarfield dark-energy models in a manner under which the two scenarios can be simultaneously valid. This type of approach is available in the cosmological literature (e.g., [51-53]). We have constructed the potentials and the scalar fields of these models. We observed that $\frac{\mathrm{d} V}{\mathrm{~d} z}>0$ for all of the reconstructed scalar field models up to $z \approx-0.5$ and, at very late stage (i.e., $z<-0.5$ ), we have $\frac{\mathrm{d} V}{\mathrm{~d} z}<0$. Moreover, $\frac{\mathrm{d} V}{\mathrm{~d} \phi}<0$ for all of the models.

In summary, by generalizing the previous works [51-53] to the NHDE model with $\rho_{D}=\frac{3 \phi^{2}}{4 \omega}\left(\mu H^{2}+v \dot{H}\right)$ in the framework of chameleon Brans-Dicke cosmology, we have obtained the evolution of the EoS. Following [51,92] we have considered $V, \phi$, and $f$ in power-law form and accordingly reconstructed the Hubble parameter. This approach differs from [51] in the sense that instead of considering BransDicke cosmology, we have considered chameleon BransDicke with the coupling function $f$. We have tested the SEC and WEC conditions and interpreted the evolution of the EoS from them. With some choice of the model parameters we computed the EoS and found that the computed values of the EoS are consistent with the observational results coming from SNeIa data, which suggests a limit of the EoS parameter: $-1.67<w<-0.62$ [103]. Subsequently we examined the stability for the obtained solutions of the crossing of the phantom divide under a quantum correction of massless conformally invariant fields and we have seen that quantum correction could be small when the phantom crossing occurs and the obtained solutions of the phantom crossing could be stable under the quantum correction. In the subsequent phase, we have established a correspondence between the NHDE model and the quintessence, the DBI-essence and the tachyon scalar-field models in the framework of chameleon Brans-Dicke cosmology. We reconstruct the potentials and the dynamics for these three scalar-field models we have considered. The reconstructed potentials are found to increase with evolution of the universe and in a very late stage they are observed to decay. It is also observed through the $f(\varphi)-$ $V(\varphi)$ plot that the potential is increasing with $f$, which indi- 
cates that the potential increases as the matter-chameleon coupling gets stronger with evolution of the universe.

Acknowledgments Sincere thanks are due to the anonymous reviewer for constructive suggestions. Project Grant of DST, Govt. of India No. SR/FTP/PS-167/2011 is duly acknowledged by the first author. Also, the first authors acknowledges the facilities provided by IUCAA, Pune, India, where a major portion of the work was carried out during a scientific visit in December 2013-January 2014

Open Access This article is distributed under the terms of the Creative Commons Attribution License which permits any use, distribution, and reproduction in any medium, provided the original author(s) and the source are credited.

Funded by $\mathrm{SCOAP}^{3}$ / License Version CC BY 4.0.

\section{References}

1. A.G. Riess et al., Astron. J. 116, 1009 (1998). doi:10.1086/300499

2. S. Perlmutter, Astrophys. J. 517, 565 (1999). doi:10.1086/307221

3. R.A. Knop et al., Astrophys. J. 598, 102 (2003). doi:10.1086/ 378560

4. D.N. Spergel et al., Astrophys. J. Suppl. 148, 175 (2003). doi:10. $1086 / 377226$

5. E. Komatsu et al., Astrophys. J. Suppl. 192, 18 (2011). doi:10. 1088/0067-0049/192/2/18

6. Planck Collaboration, P.A.R. Ade, N. Aghanim et al. (2013). arXiv: 1303.5076

7. D.J. Eisenstein et al. (SDSS Collaboration), Astrophys. J. 633, 560 (2005). doi:10.1086/466512

8. W.J. Percival et al., Mon. Not. R. Astron. Soc. 401, 2148 (2010). doi:10.1111/j.1365-2966.2009.15812.x

9. E.J. Copeland, M. Sami, S. Tsujikawa, Int. J. Mod. Phys. D 15, 1753 (2006). doi:10.1142/S021827180600942X

10. K. Bamba, S. Capozziello, S. Nojiri, S.D. Odintsov, Astrophys. Space Sci. 342, 155 (2012). doi:10.1007/s10509-012-1181-8

11. R.R. Caldwell, M. Kamionkowski, Ann. Rev. Nucl. Part. Sci. 59, 397 (2009). doi:10.1146/annurev-nucl-010709-151330

12. S. Nojiri, S.D. Odintsov, Phys. Rep. 505, 59 (2011). doi:10.1016/ j.physrep.2011.04.001

13. T. Clifton, P.G. Ferreira, A. Padilla, C. Skordis, Phys. Rep. 513, 1 (2012). doi:10.1016/j.physrep.2012.01.001

14. S. Capozziello, M. De Laurentis, S.D. Odintsov, Eur. Phys. J. C 72, 2068 (2012). doi:10.1140/epjc/s10052-012-2068-0

15. S. Tsujikawa, Lect. Notes Phys. 800, 99 (2010). doi:10.1007/ 978-3-642-10598-2 3

16. V. Sahni, A.A. Starobinsky, Int. J. Mod. Phys. D 9, 373 (2000). doi:10.1142/S0218271800000542

17. V. Sahni, A.A. Starobinsky, Int. J. Mod. Phys. D 15, 2105 (2006). doi:10.1142/S0218271806009704

18. H. Motohashi, A.A. Starobinsky, J. Yokoyama, Prog. Theor. Phys. 123, 887 (2010). doi:10.1143/PTP.124.541

19. P.J.E. Peebles, B. Ratra, Rev. Mod. Phys. 75, 559 (2003). doi:10. 1103/RevModPhys.75.559

20. A. Shafieloo, V. Sahni, A.A. Starobinsky, Phys. Rev. D 80, 101301 (2009). doi:10.1103/PhysRevD.80.101301

21. A.A. Starobinsky, Phys. Lett. B 91, 99 (1980). doi:10.1016/ 0370-2693(80)90670-X

22. S. Nojiri, S.D. Odintsov, O.G. Gorbunova, J. Phys. A Math. Gen. 39, 6627 (2006). doi:10.1088/0305-4470/39/21/S62

23. A.V. Astashenok, S. Nojiri, S.D. Odintsov, R.J. Scherrer, Phys. Lett. B 713, 145 (2012). doi:10.1016/j.physletb.2012.06.017
24. B. Gumjudpai, T. Naskar, M. Sami, S. Tsujikawa, J. Cosmol. Astropart. Phys. 06, 007 (2005). doi:10.1088/1475-7516/2005/ 06/007

25. E. Elizalde, S. Nojiri, S.D. Odintsov, Phys. Rev. D 70, 043539 (2004). doi:10.1103/PhysRevD.70.043539

26. S. Nojiri, S.D. Odintsov, S. Tsujikawa, Phys. Rev. D 71, 063004 (2005). doi:10.1103/PhysRevD.71.063004

27. H. Zhang, Z-H. Zhu. Phys. Rev. D 73, 043518 (2006). doi:10. 1103/PhysRevD.73.043518

28. K. Bamba, J. Matsumoto, S. Nojiri, Phys. Rev. D 85, 084026 (2012). doi:10.1103/PhysRevD.85.084026

29. M. Forte, Phys. Rev. D 90, 027302 (2014). doi:10.1103/ PhysRevD.90.027302

30. M. Li, Phys. Lett. B 603, 1 (2004). doi:10.1016/j.physletb.2004. 10.014

31. E. Elizalde, S. Nojiri, S.D. Odintsov, P. Wang, Phys. Rev. D 71, 103504 (2005). doi:10.1103/PhysRevD.71.103504

32. S. Nojiri, S.D. Odintsov, Gen. Relativ. Gravit. 38, 1285 (2006). doi:10.1007/s10714-006-0301-6

33. S. del Campo, J.C. Fabris, R. Herrera, W. Zimdahl, Phys. Rev. D 83, 123006 (2011). doi:10.1103/PhysRevD.83.123006

34. J.-L. Cui, J.-F. Zhang, Eur. Phys. J. C 74, 2849 (2014). doi:10. 1140/epjc/s10052-014-2849-8

35. Q.-G. Huang, Y. Gong, JCAP 0408, 006 (2004). doi:10.1088/ 1475-7516/2004/08/006

36. Q.-G. Huang, M. Li, JCAP 0503, 001 (2005). doi:10.1088/ 1475-7516/2005/03/001

37. X. Zhang, F-Q. Wu. Phys. Rev. D 76, 023502 (2007). doi:10.1103/ PhysRevD.76.023502

38. Y.-F. Cai, E.N. Saridakis, M.R. Setare, J.-Q. Xia, Phys. Rep. 493, 1 (2010). doi:10.1016/j.physrep.2010.04.001

39. A.A. Starobinsky, Phys. Rev. Lett. 85, 2236 (2000). doi:10.1103/ PhysRevLett.85.2236

40. S. Nojiri, S.D. Odintsov, Phys. Lett. B 562, 147 (2003). doi:10. 1016/S0370-2693(03)00594-X

41. S. Nojiri, S.D. Odintsov, Phys. Lett. B 571, 1 (2003). doi:10.1016/ j.physletb.2003.08.013

42. S. Nojiri, S.D. Odintsov, Phys. Lett. B 565, 1 (2003). doi:10.1016/ S0370-2693(03)00753-6

43. I. Brevik, S. Nojiri, S.D. Odintsov, L. Vanzo, Phys. Rev. D 70, 043520 (2004). doi:10.1103/PhysRevD.70.043520

44. S. Nojiri, S.D. Odintsov, Proc. Sci. WC 2004, 024 (2004). arXiv:hep-th/0412030

45. B. Novosyadlyj, O. Sergijenko, R. Durrer, V. Pelykh, JCAP (2013). doi:10.1088/1475-7516/2013/06/042

46. M.R. Setare, E.N. Saridakis, Phys. Rev. D 79, 043005 (2009). doi:10.1103/PhysRevD.79.043005

47. H. Wei, R.-G. Cai, D.-F. Zeng. Class. Quantum Gravity 22, 3189 (2005). doi:10.1088/0264-9381/22/16/005

48. S. Nojiri, S.D. Odintsov, Phys. Lett. B 716, 377 (2012). doi:10. 1016/j.physletb.2012.08.049

49. S. Nojiri, S.D. Odintsov, N. Shirai, J. Cosmol. Astropart. Phys. 1305, 020 (2013). doi:10.1088/1475-7516/2013/05/020

50. K. Bamba, S. Nojiri, S. D. Odintsov, Report OCHA-PP-323 (2014). arXiv:1406.2417 [hep-th]

51. W. Yang, Y. Wu, L. Song, Y. Su, J. Li, D. Zhang, X. Wang, Mod. Phys. Lett. A 26, 191 (2011). doi:10.1142/S0217732311034682

52. K. Karami, J. Fehri, Phys. Lett. B 684, 61 (2010). doi:10.1016/j. physletb.2009.12.060

53. A. Sheykhi, Phys. Lett. B 681, 205 (2009). doi:10.1016/j.physletb. 2009.10.011

54. L.N. Granda, A. Oliveros, Phys. Lett. B 669, 275 (2008). doi:10. 1016/j.physletb.2008.10.017

55. M. Li, X.-D. Li, J. Meng, Z. Zhang, Phys. Rev. D 88, 023503 (2013). doi:10.1103/PhysRevD.88.023503 
56. L.N. Granda, A. Oliveros, Phys. Lett. B 671, 199 (2009). doi:10. 1016/j.physletb.2008.12.025

57. M. Sharif, A. Jawad, Eur. Phys. J. C 72, 2097 (2012). doi:10.1140/ epjc/s10052-012-2097-8

58. A. Jawad, S. Chattopadhyay, A. Pasqua, Astrophys. Space Sci. 346, 273 (2013). doi:10.1007/s10509-014-2010-z

59. M. Malekjani, A. Khodam-Mohammadi, N. Nazari-pooya, Astrophys. Space Sci. 332, 515 (2011). doi:10.1007/ s10509-010-0550-4

60. U. Debnath, S. Chattopadhyay, Int. J. Theor. Phys. 52, 1250 (2013). doi:10.1007/s10773-012-1440-z

61. S. Nojiri, S.D. Odintsov, J. Phys. Conf. Ser. 66, 012005 (2007). doi:10.1088/1742-6596/66/1/012005

62. S. Nojiri, S.D. Odintsov, Int. J. Geom. Methods Mod. Phys. 4(115), 2007. doi:10.1142/S0219887807001928. [eConf C0602061 (2006) 06]

63. S. Nojiri, S.D. Odintsov, Int. J. Geom. Methods Mod. Phys. 11, 1460006 (2014). doi:10.1142/S0219887814600068

64. S. Capozziello, V.F. Cardone, V. Salzano, Phys. Rev. D 78, 063504 (2008). doi:10.1103/PhysRevD.78.063504

65. K. Bamba, C-Q. Geng, C-C. Lee. Int. J. Mod. Phys. D 20, 1339 (2011). doi:10.1142/S0218271811019517

66. K. Bamba, C-Q. Geng. Prog. Theor. Phys. 122, 1267 (2009). doi:10.1143/PTP.122.1267

67. R. Myrzakulov, Eur. Phys. J. C 71, 1752 (2011). doi:10.1140/epjc/ s10052-011-1752-9

68. K. Bamba, C-Q. Geng, C-C. Lee, L-W. Luo, JCAP 1101, 021 (2011). doi:10.1088/1475-7516/2011/01/021

69. K. Bamba, Y. Kokusho, S. Nojiri, N. Shirai, Class. Quantum Gravity 31, 075016 (2014). doi:10.1088/0264-9381/31/7/075016

70. Y. Ito, S. Nojiri, S.D. Odintsov, Entropy 14, 1578 (2012). doi:10. 3390/e14081578

71. K. Nozari, A. Behboodi, S. Akhshabi, Phys. Lett. B 723, 201 (2013). doi:10.1016/j.physletb.2013.04.058

72. A. Ali, R. Gannouji, M. Sami, Phys. Rev. D 82, 103015 (2010). doi:10.1103/PhysRevD.82.103015

73. K. Bamba, A.N. Makarenko, A.N. Myagky, S.D. Odintsov, Phys. Lett. B 732, 349 (2014). doi:10.1016/j.physletb.2014.04.004

74. K. Bamba, S. Nojiri, S.D. Odintsov, JCAP 0810, 045 (2008). doi:10.1088/1475-7516/2008/10/045

75. K. Bamba, C.-Q. Geng, S. Nojiri, S.D. Odintsov, Phys. Rev. D 79, 083014 (2009). doi:10.1103/PhysRevD.79.083014

76. K. Bamba, R. Myrzakulov, S. Nojiri, S.D. Odintsov, Phys. Rev. D 85, 104036 (2012). doi:10.1103/PhysRevD.85.104036

77. K. Bamba, C.-Q. Geng, C.-C. Lee, JCAP 1008, 021 (2010). doi:10.1088/1475-7516/2010/08/021

78. K. Bamba, in Proceedings of the KMI Inauguration Conference, Nagoya University, Nagoya, Japan, 24-26 October 2011 (2011). arXiv: 1202.4317 [gr-qc]

79. C. Brans, R.H. Dicke, Phys. Rev. 124, 925 (1961). doi:10.1103/ PhysRev.124.925

80. K. Bamba, D. Momeni, R. Myrzakulov (2014) arXiv:1404.4255 [hep-th]

81. M. Jamil, D. Momeni, Chin. Phys. Lett. 28, 099801 (2011). doi:10. 1088/0256-307X/28/9/099801

82. M. Jamil, D. Momeni, M.A. Rashid, Eur. Phys. J. C 71, 1711 (2011). doi:10.1140/epjc/s10052-011-1711-5
83. M. Jamil, I. Hussain, D. Momeni, Eur. Phys. J. Plus 126, 80 (2011). doi:10.1140/epjp/i2011-11080-2

84. M. Jamil, D. Momeni, M. Raza, R. Myrzakulov, Eur. Phys. J. C 72, 1999 (2012). doi:10.1140/epjc/s10052-012-1999-9

85. D. Momeni, M.R. Setare, Mod. Phys. Lett. A 26, 2889 (2011) doi:10.1142/S0217732311037169

86. S. Sen, T.R. Seshadri, Int. J. Mod. Phys. D 12, 445 (2003). doi: 10. 1142/S0218271803003049

87. H. Alavirad, A. Sheykhi, Phys. Lett. B. 734, 148 (2014). doi:10. 1016/j.physletb.2014.05.023

88. J. Khoury, A. Weltman, Phys. Rev. D 69, 044026 (2004). doi:10. 1103/PhysRevD.69.044026

89. J. Khoury, A. Weltman, Phys. Rev. Lett. 93, 171104 (2004) doi:10.1103/PhysRevLett.93.171104

90. T. P. Waterhouse (2006). astro-ph/0611816

91. S. Chattopadhyay, ISRN High Energy Phys. 2013, 414615 (2013). doi:10.1155/2013/414615

92. Y. Bisbar, Phys. Rev. D 86, 127503 (2012). doi:10.1103/ PhysRevD.86.127503

93. R.A. El-nabulsi, Eur. Phys. J. Plus 127, 23 (2012). doi:10.1140/ epjp/i2013-13055-7

94. A. Upadhye, S.S. Gubser, J. Khoury, Phys. Rev. D 74, 104024 (2006). doi:10.1103/PhysRevD.74.104024

95. S.S. Gubser, J. Khoury, Phys. Rev. D 70, 104001 (2004). doi:10. 1103/PhysRevD.70.104001

96. S. Chattopadhyay, U. Debnath, Int. J. Mod. Phys. D 20, 1135 (2011). doi:10.1142/S0218271811019293

97. H. Farajollahi, A. Salehi, Phys. Rev. D 85, 083514 (2012). doi:10. 1103/PhysRevD.85.083514

98. Kh Saaidi, A. Mohammadi, T. Golanbari, H. Sheikhahmadi, B. Ratra, Phys. Rev. D 86, 045007 (2012). doi:10.1103/PhysRevD. 86.045007

99. A. Pasqua, S. Chattopadhyay, Astrophys. Space Sci. 348, 283 (2013). doi:10.1007/s10509-013-1557-4

100. E.N. Saridakis, Nucl. Phys. B 819, 116 (2009). doi:10.1016/j. nuclphysb.2009.04.011

101. M. Yashar, B. Bozek, A. Abrahamse, A. Albrecht, M. Barnard, Phys. Rev. D 79, 103004 (2009). doi:10.1103/PhysRevD.79. 103004

102. O. Hrycyna, M. Szydowski, Phys. Rev. D 88, 064018 (2013). doi:10.1103/PhysRevD.88.064018

103. A.A. Usmani, P.P. Ghosh, U. Mukhopadhyay, P.C. Ray, S. Ray, Mon. Not. R. Astron. Soc. 386, L92 (2008). doi:10.1111/j. 1745-3933.2008.00468.x

104. K. Bamba, G. Cognola, S.D. Odintsov, S. Zerbini, Phys. Rev. D 90, 023525 (2014). doi:10.1103/PhysRevD.90.023525

105. P. J. Steinhardt, Philos. Trans. R. Soc. Lond. A 361, 2497 (2003). doi:10.1098/rsta.2003.1290

106. J. Martin, M. Yamaguchi, Phys. Rev. D 77, 123508 (2008). doi:10. 1103/PhysRevD.77.123508

107. T. Padmanabhan, Phys. Rev. D 66, 021301 (2002). doi:10.1103/ PhysRevD.66.021301

108. J.S. Bagla, H.K. Jassal, T. Padmanabhan, Phys. Rev. D 67, 063504 (2003). doi:10.1103/PhysRevD.67.063504 